American J. of Engineering and Applied Sciences 2 (2): 466-470, 2009

ISSN 1941-7020

(C) 2009 Science Publications

\title{
Basic Theoretical Backgrounds of the Engineering Design Procedure of a Drying Plant
}

\author{
Jamil Sami Haddad \\ Design and Manufacturing Division, Mechanical Design and Technology Centre, \\ Royal Scientific Society, P.O. Box 1438 Al-Jubaiha 11941 Jordan
}

\begin{abstract}
Problem statement: This study was made to reduce energy consumption and increase productivity of drying process in many industries, to achieve the required aim, design arithmetic methods and choose the parameters of the drying machine and computing additional factors including characteristics of materials and air flow effect. Approach: The design of these types of dryers did not include the addressed factors during the design; the study of these new factors will provide better understanding of the process and increase the abilities for improvement. study made to define the engineering design procedure of a material drying plant which include the size (length, width, height) of the operating member, amplitude and vibration frequency that provide necessary productivity, efficient parameters of material and air movement, necessary drying time and to specify quantity and condition of material subjected to drying. Results: The improvement included the conveyer design which consisted of shutters; the shutter design includes size, angle of inclination and gab size. After applying primary data and the standard thermo computation, the dynamic parameters are set and then the geometric parameters. According to the mathematical computation the design was made and then the verifying of the elements of speed of material motion, air current and the wetness of material was built accordingly. The result of this study was mathematical method for defining the dimensions of drying machine to obtain optimum productivity with lowest energy consumption. These mathematical equations can solve the problem of low productivity and high power consumption if implemented correctly and taking into consideration the dynamic and geometric elements of each case. Conclusion: The results of this study can be implemented in many fields of the industry where dryers were used, the application of the mathematical relations can improve the revenue by increasing productivity and lowering power consumption for industry. For further study, the study of thermodynamics on the movement of particles will be advised.
\end{abstract}

Key words: Vibrator, conveyor, dryer, energy, productivity, shutter

\section{INTRODUCTION}

The process of drying extracted materials is considered one of the most important preparatory and final stages of separating raw materials process.

It is known that any process involving water removing consumes less energy compared with the dry processes. In the mean time, the processes of removing water is not designed to remove humidity, this can only be obtained by the drying processes.

Materials drying can be done directly in crushers or grinders or in independent drying machines. The economical cost of drying processes depends on many factors such as the period of the materials presence in the drying passage, temperature degree, ratio of wetness, size of granules, the space of contact between the dryer and materials, also the type of contact with a solid object or gas-dynamics ${ }^{[1]}$.
Experience shows that using the vibrating drying machines with pumping hot air through the vibrating hot layer conceder as a high economic and efficient method for drying materials.

The vibrating drying machine (Fig. 1) consists of a framework that is equipped with a compact passage (leakage resistant) and divided by the gas distribution jalousie into two parts. The feeding section is fixed to one end of the passage and the evacuation section is fixed to the other end.

The operating surface of the feeding section is divided into two sections: upper and lower. The upper section is attached to a feeding regulator; whereas, the lower section is attached to the gas burner equipped with pumping fan to provide hot air.

At the last section of the passage, a suction pipe is attached for the transportation, of air, volatiles granules in to a cyclone using a powerful fan. 


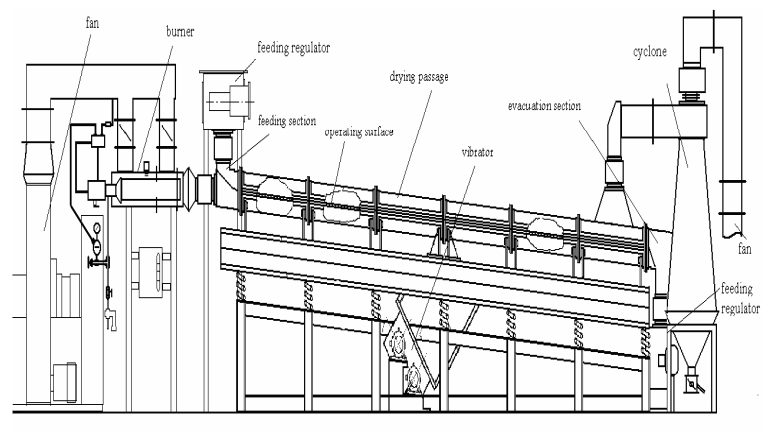

Fig. 1: The vibrating drying unit

The framework is based on the brackets of vibration dampers springs. The vibration motion is created by a controlled instigator.

It is possible to increase the efficiency of the vibrating conveyor by designing arithmetic methods, choice of the dynamic and designing parameters with computing the extra factors, related to the existence of air currents and the change of the characteristics of materials that affect the nature of moving them on the operating element at the time of drying.

\section{MATERIALS AND METHODS}

Part of the initial data for the suggested technology is obtained from the design made according to the special instruction of ITM of National Academy of Science of Ukraine. Let us define the initial data for the calculations according to the given instruction:

- Output in solid product, $\mathrm{Q}, \mathrm{t} / \mathrm{h}$

- Initial humidity of the product, $\mathrm{W}_{\mathrm{I}}, \%$

- End humidity of the product, $\mathrm{W}_{\mathrm{E}}, \%$

- Characteristic of the product (granulometric composition, specific heat capacity and maximum heating temperature)

- Composition and characteristic of the heat-transfer medium (chemical composition and relative humidity)

On the basis of heat calculations of the product taking into account working peculiarities of vibratory drying plant of the given project the temperature $\mathrm{T}^{\circ} \mathrm{K}$ of the heat-transfer medium and necessary drying time $t_{c}$ is defined (technology of ITM of National Academy of Science of Ukraine).

According to our engineering technology the following parameters of drying plant of the vibrating conveyor are defined:
- Length L, width $\mathrm{B}$, slot size $\mathrm{h}$, inclination angle yand width $b$ of the shutters plates, height of the bottom part of the operating member $\mathrm{H}_{\mathrm{b}}$, height of the upper part of the operating member $\mathrm{H}_{\mathrm{u}}$

- Average velocity of the vibratory displacement of the material $\mathrm{v}_{\mathrm{a}}$

- Stiffness characteristic of the extended operating member of the vibrating drying plant

- Amplitude a and vibration frequency $\omega$ of the operating member, angle $\beta$ of the vibration direction

- Stiffness C of the elastic elements of vibrating conveyor, kinetostatic moment $\mathrm{m}_{0} \mathrm{r}$ of unbalance weight of the vibroexciter

Granulometric composition of the product is defined with the help of maximum $\mathrm{D}$ and weightaverage $d_{a}$ sizes of the pieces of material.

Rate of volume productivity of the vibratory drying plant can be calculated by the following formula:

$\mathrm{V}=\frac{\mathrm{Q}}{3,6 \gamma_{\mathrm{f}}}, \mathrm{m}^{3} \mathrm{~s}^{-1}$

where, $\gamma_{\mathrm{f}}$-density of the material in filling, $\mathrm{kg} \mathrm{m}^{-3}$.

According to the known velocity of hovering of weight-average piece of the material $\mathrm{v}_{\mathrm{h}}$ we define the total area of slots of the vibratory drying plant:

$S_{s}=\frac{V_{d}}{v_{h}}$

where $\mathrm{V}_{\mathrm{d}}$ - discharge of heat-transfer medium $\left(\mathrm{m}^{3} \mathrm{~s}^{-1}\right)$.

Plates of the functional surface of the shutters of the vibrating conveyor are characterized by the following parameters: width $b$, length $B$ (equal to the width of the tray of the vibratory conveyor), width of the plate $\delta_{\mathrm{p}}$, inclination angle $\gamma$, gap size between the plates $h_{p}$, additional width area of the plate $b_{0}$, securing their overlapping (Fig. 2).

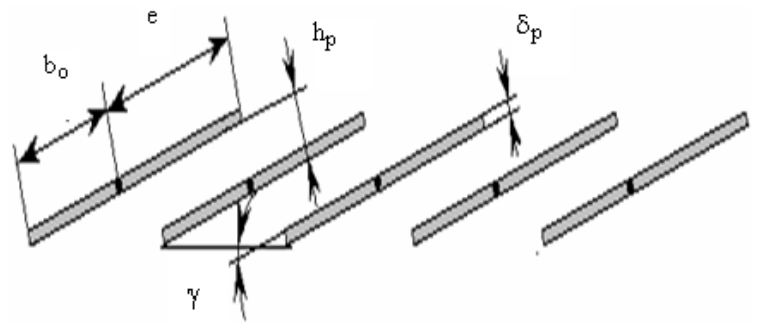

Fig. 2: Specification of parameters of the shutters opening 
Minimum inclination angle of the plates (when there is no gap between the plates):

$$
\gamma_{0}=\arcsin \left(\frac{\delta_{\mathrm{p}}}{\mathrm{b}}\right)
$$

As a result of the experimental research it has been established that rational range of values of the inclination angle of shutters $\min \leq \gamma \leq \gamma_{\max }$ is in the range of $2 \div 5^{\circ}$ (in some cases up to $7^{\circ}$ ). In the same time depending on the type of the material the variable $\gamma$ can be accepted as constant, go down to the discharge end of the vibrating conveyor (for example while sand drying), or go up (for example, while chalk drying). Rational variable of gap $h_{p}$ between the plates of he shutters is also in the range of $2 \div 5 \mathrm{~mm}$, depending on the type and granulometric composition of the material. Consequently using the accepted values of size of the gap between the plates and inclination angle of the shutters plates, their width will be defined as:

$\mathrm{b}=\frac{\mathrm{h}_{\mathrm{p} . \mathrm{a}}+\delta_{\mathrm{p}}}{\sin \left(\gamma_{\mathrm{a}}\right)}$

While material drying, it is important to provide its uniform division in width and length of the operating member. It constrains to the certain point the width of the functional surface and the distribution of inclination angle of the shutters along the operating member because these parameters are used in some range to regulate the velocity of the material movement as a result of its humidity change.

As a result of experimental research and the experience of running vibratory drying plants the following points can be recommended:

- For ordinary lump material-height of the material layer $\mathrm{h} \leq(1 \div 1,5) \mathrm{D}$ (Fig. 3), width of the tray $-\mathrm{B}=(8 \div 10) \mathrm{D}$

- For fine-dispersed material value $\mathrm{h} \leq 50 \mathrm{~mm}$, $\mathrm{B} \leq 500 \mathrm{~mm}$

Specifying the tray width of the vibratory dryer B and choosing gap average value between the plates $h_{p . a}$, number of plates will be defined as:

$$
\mathrm{p}_{\mathrm{n}}=\frac{\mathrm{S}_{\mathrm{s}}}{\mathrm{Bh}_{\mathrm{p} . \mathrm{a}}}
$$

and the length of the conveyor:

$$
\mathrm{L}=\mathrm{bp} \mathrm{p}_{\mathrm{n}}
$$

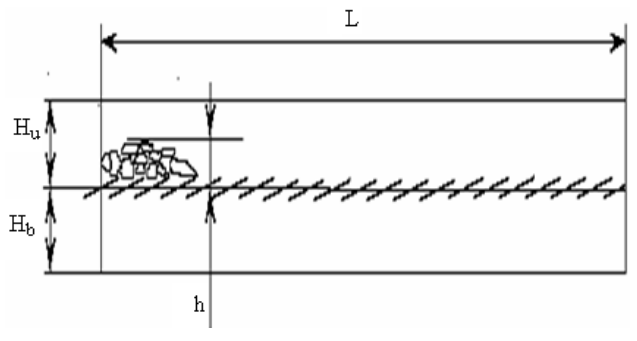

Fig. 3: Specification of length of the conveyor according to the area of the shutters gaps

Next average velocity of vibratory displacement of the material is defined:

$\mathrm{v}_{\mathrm{a}}=\frac{\mathrm{L}}{\mathrm{t}_{\mathrm{c}}}$

The average velocity of vibratory displacement of the material is compared with the rational values for vibratory units $\left(0,01 \leq \mathrm{v}_{\mathrm{a}} \leq 0,2 \mathrm{~m} \mathrm{~s}^{-1}\right)$. If necessary the length of the conveyor, number (or width) of the plates are corrected. Work is also done to verify the chosen parameters of vibrating conveyor from the point of view of providing specified productivity according to the expression:

$\mathrm{V}=\mathrm{Bhv}_{\mathrm{a}}$

If necessary the tray width of the conveyor is corrected as:

$\mathrm{B}=\frac{\mathrm{V}}{\mathrm{hv}_{\mathrm{a}}}$

With the corresponding correction of the slot size between the plates $\mathrm{h}_{\text {p.a }}$.

Inclination angle of the operating member to the level $\alpha$ is usually chosen to be equal to the maximum angle of setting of the shutters plates. Angle of vibration direction, required amplitude extent and oscillation frequency of the operating member is found from the criterion of providing necessary velocity of vibratory displacement of the material ${ }^{[2-4]}$. Here it should be taken into account that for lump materials it is usual to use the movement mode with separation from the surface while for fine-dispersed materials movement mode without separation from the functional surface.

Height of trays under the functional surface and above the layer of the material is defined considering the following: 
Height of the underpan (the tray above the functional surface) (Fig. 3) is found at the condition of providing the required velocity of the initial current of heat-transfer medium. As a result of the experimental research it has been established that there will be normal washing of the material layer with the flow (without any swirling and backflows). If the velocity of the supplied flow of heat-transfer medium does not exceed $v_{h}=10 \mathrm{~m} \mathrm{~s}^{-1[5]}$, then the height of the underpan is:

$$
\mathrm{H}_{\mathrm{b}}=\frac{\mathrm{V}_{\mathrm{d}}}{\mathrm{B} \cdot \mathrm{v}_{\mathrm{h}}}
$$

Height of trays above the functional surface is defined considering the velocity of hovering of the particles of maximum permissible size $d_{e}$ (maximum size of the particle which drying could be completed within the time of being in the air flow). Productivity of the plant as for outgoing flow $\mathrm{V}_{\text {d.o }}$ is defined as the productivity on supplied heat-transfer medium taking into account volume of evaporated moisture. The quantity of evaporated moisture in unit time is defined as:

$\mathrm{Q}_{\mathrm{e}}=\frac{\mathrm{Q}\left(\mathrm{W}_{\mathrm{I}}-\mathrm{W}_{\mathrm{E}}\right)}{360}, \mathrm{~kg} \mathrm{~s}^{-1}$

That is in volume terms from ${ }^{[6,7]}$ :

$\mathrm{V}_{\mathrm{e}}=\frac{1537}{\gamma_{\mathrm{e}}} \mathrm{Q}_{\mathrm{e}}, \mathrm{m}^{3} \mathrm{~s}^{-1}$

where $\gamma_{\mathrm{e}}$ - density of evaporated moisture (water).

Total productivity of the plant on the outgoing flow of heat-transfer medium:

$\mathrm{V}_{\text {d.o }}=\mathrm{V}_{\mathrm{d}}+\mathrm{V}_{\mathrm{e}}$

Then at the known velocity of hovering of particles of maximum permissible size $\mathrm{v}_{\text {e.p }}$, height of the tray above the functional surface will be:

$$
\mathrm{H}_{\mathrm{u}}=\frac{\mathrm{V}_{\mathrm{d} . \mathrm{o}}}{\mathrm{Bv}_{\text {e.p }}}+\mathrm{h}
$$

While doing dynamic calculations of vibrating conveyor, the important parameter is the quantity of the material on the functional surface.

Mass of the material on the functional surface consists of the initial material mass, except the material being removed by the flow of heat-transfer medium and moisture being transported along with the material. Relative quantity of moisture in the material is defined with its initial $\mathrm{W}_{\mathrm{I}}$ and final $\mathrm{W}_{\mathrm{E}}$ values. Accepting the law of variation of humidity along the tray of conveyor to be exponential, we get the expression for the material humidity in any site of the operating member as:

$\mathrm{W}=\mathrm{W}_{\mathrm{I}} \exp \left(\frac{\mathrm{x}}{\mathrm{L}}\right) \ln \frac{\mathrm{W}_{\mathrm{E}}}{\mathrm{W}_{\mathrm{I}}}$

The average value of humidity along the operating member is defined as:

$$
\mathrm{W}_{\mathrm{a}}=\frac{1}{\mathrm{~L}} \int_{0}^{\mathrm{L}} \mathrm{Wdx}
$$

Or, after substitution $\mathrm{W}$, integration and some transformations we get:

$$
\mathrm{W}_{\mathrm{a}}=\frac{\mathrm{W}_{\mathrm{I}}}{\ln \frac{\mathrm{W}_{\mathrm{I}}}{\mathrm{W}_{\mathrm{E}}}}\left[1-\exp -\left(\ln \frac{\mathrm{W}_{\mathrm{I}}}{\mathrm{W}_{\mathrm{E}}}\right)\right]
$$

Considering all the aforesaid, the mass of the material on the functional surface can be defined as:

$$
\mathrm{M}_{\mathrm{m}} \cong \mathrm{QL}\left(1+\frac{\mathrm{W}_{\mathrm{a}}}{100}\right)\left(1-\alpha_{\mathrm{y}}\right) /\left(2 \mathrm{v}_{\mathrm{a}}\right)
$$

Where:

$\mathrm{Q}=$ Efficiency $\left(\mathrm{kg} \mathrm{s}^{-1}\right)$

$\mathrm{L}=$ Length of tray (m)

$\alpha_{y}=$ Part of material, being removed with heat-transfer medium

$\mathrm{v}_{\mathrm{a}}=$ Average velocity of vibrotransportation $\left(\mathrm{m} \mathrm{s}^{-1}\right)$

Added mass of the material is defined as:

$\mathrm{M}_{\text {add. }}=\mathrm{k}_{\text {add. }} \mathrm{M}_{\mathrm{m}}$

where, $\mathrm{k}_{\mathrm{add}}$-coefficient of addition which depends on the mode of vibratory displacement $\Gamma_{1}{ }^{[2-4]}$ and defined from empirical dependence as:

$$
\mathrm{k}_{\text {add. }}=0,73 / \Gamma_{1}-0,18
$$

Weight of tray considering the mass of the material is defined as:

$\mathrm{M}_{1}=\mathrm{M}_{\mathrm{t}}+\mathrm{M}_{\text {add. }}$ 
This value is taken to be basic while choosing the parameters of vibroexciter and elastic system of machine.

Further, after accomplishing engineering study and defining the weight of the working member, parameters of elastic system and vibroexiter are defined.

\section{RESULTS AND DISCUSSION}

The developed engineering technology due to the numerous influencing factors provides iterative process of calculation and parameters selection of the vibratory conveyor of drying plant. At the first stage works are done to define size (length, width, height) of the operating member, amplitude and its vibration frequency that provide necessary productivity, efficient parameters of material and wind movement, necessary drying time and to get some specifications as for quantity and condition of material subjected to drying. After the engineering study, improved calculation is done taking into consideration set of factors influencing conveyor dynamics and process of vibratory displacement of the material. Besides alignment of the parameters of vibratory conveyor are done.

\section{CONCLUSION}

The received analytical dependences and the introduced procedure of calculations and choosing the parameters allow to choose the parameters of vibrating conveyor of vibratory drying plant as a mechanical system considering the influence of the material subjected to drying and heat-transfer medium.

\section{REFERENCES}

1. Haddad, J.S., 2004. The laboratory model of the vibrating drying machine for experimental studies of its working parameters. Sci. Herald NMU., 9: 58-60.

2. Haddad, J.S., 2005. The transporting of raw materials in the vibrating drying machine. Scientific Herald NMU., 2: 45-47.

3. Franchuk, V.P., V.A. Fidoskin and J.S. Haddad, 2005. Defining the motion speed of the materials with computing the effect of the bend's oscillation of the operating element of the vibrating drying machine. Sci. Herald NMU., 12: 78-80.

4. Franchuk, V.P., V.A. Fidoskin and J.S. Haddad, 2004. The effect of the bend's oscillation on the motion of materials in the vibrating drying machine. Vibrat. Equip. Technol., 1: 80-83.

5. Franchuk, V.P. and J.S. Haddad, 2005. Defining the characteristic of air motion in the vibrating drying machine. Proceeding of the International Conference on Improvement of the Quality Reliability and Long Usage of Technical Systems and Technological Processes, Dec. 4-11, Egypt, pp: 74-78.

6. Abramovich G.N., 1969. Applied Gas Dynamics. M. Science, pp: 824.

7. Litvin A.M., 1969. Theoretical Basis of Thermal Engineering. M. Energy, pp: 328. 